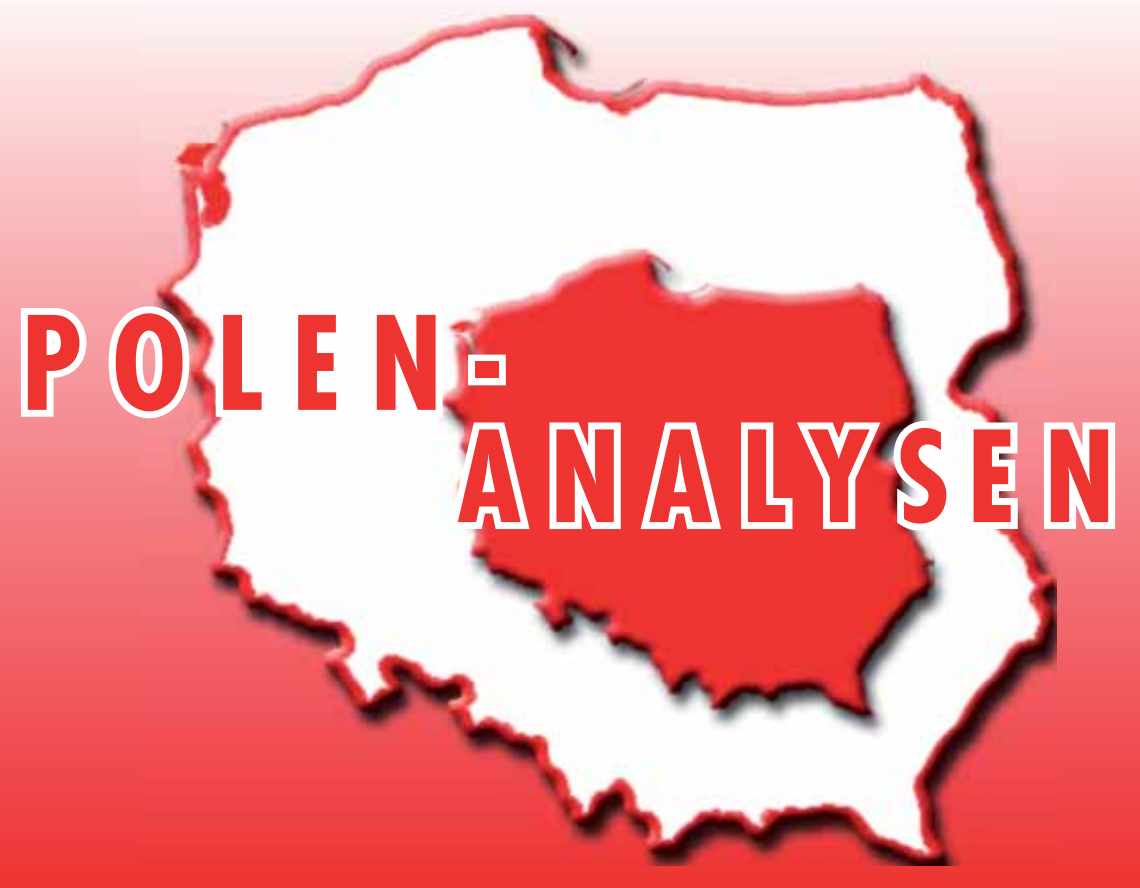

www.laender-analysen.de/polen

\title{
EIN JAHR REGIERUNG TUSK
}

aNALYSE

Ein Jahr Regierung Tusk

Janusz A. Majcherek, Krakau

- TABELLEN UND GRAFIKEN

Die Regierung Tusk im Spiegel von Meinungsumfragen

CHRONIK

Vom 18. November bis zum 01. Dezember 2008

Die nächste Ausgabe der Polen-Analysen erscheint nach der Weihnachtspause am 20. Januar 2009.

Die Redaktion der Polen-Analysen wünscht ihren Leserinnen und Lesern ein frohes Weihnachtsfest und ein gutes, gesundes und erfolgreiches Jahr 2009!

Die Herausgeber danken der BSH Bosch und Siemens Hausgeräte GmbH München und dem Stifterverband für die Deutsche Wissenschaft für ihre Unterstützung. 


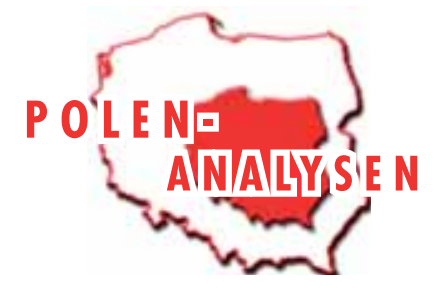

Analyse

\section{Ein Jahr Regierung Tusk}

Janusz A. Majcherek, Krakau

Eine Bilanz des ersten Jahrs der Regierung von Ministerpräsident Donald Tusk erfordert eine differenzierte Analyse der Programme und Entscheidungen einerseits und des Regierungsstils andererseits, wobei die beiden Aspekte gleichwertig betrachtet werden sollten. Dieser Zugang zum Thema wird zum einen durch die Umstände und Gründe für den Wahlsieg der Bürgerplattform (Platforma Obywatelska - PO) im Herbst 2007 und zum anderen durch die nach wie vor für polnische Verhältnisse ungewöhnlich hohe gesellschaftliche Zustimmung zu einer Regierungspartei nach einem Jahr Regierungszeit nahe gelegt. Die Partei des Ministerpräsidenten, die Bürgerplattform, hat fast doppelt so viele Anhänger wie die nächstgrößte Partei, die oppositionelle Recht und Gerechtigkeit (Prawo i Sprawiedliwość - PiS). Dagegen kann sie für wichtige Reformvorhaben nicht auf die Unterstützung des Staatspräsidenten, der Oppositionsparteien oder der Gewerkschaften zählen.

$\mathrm{V}^{\mathrm{s}}$ or den vorgezogenen Parlamentswahlen im vergangenen Jahr kam es zu einer beispiellosen Mobilisierung der jungen, gut ausgebildeten, in großen Städten lebenden gesellschaftlichen Gruppen, die mit ihren Stimmen über die Abwahl der kaum zwei Jahre lang regierenden Partei der Brüder Kaczyński Recht und Gerechtigkeit (Prawo i Sprawiedliwość - PiS) entschieden. Der Anstieg der Wahlbeteiligung und die große Abneigung gegenüber der Regierung lag vor allem im Regierungsstil ihrer führenden Köpfe begründet. Dieser drückte sich in der Polarisierung der Gesellschaft aus, im Schüren innergesellschaftlicher Konflikte, in der wachsenden Aggression gegenüber politischen und ideologischen Gegnern, der Initiierung immer neuer Kampagnen gegen tatsächliche und virtuelle Feinde und in zur Schau gestellten Animositäten gegenüber anderen Nationen, darunter auch bzw. insbesondere gegenüber Deutschland. Die zweijährige Phase einer Politik des Kampfes vor allem gegen Personen, der unter dem Schlachtruf einer moralischen Revolution geführt wurde, der Bruch mit dem bisherigen Modell der polnischen Transformation und die Proklamierung der IV. Republik rief in der Gesellschaft Überdruss, Ermüdung und schließlich Empörung hervor, die in den Wahlen abreagiert wurden. Ihr Ergebnis war in höheremAusmaß Ausdruck der Ablehnung der PiS als der Unterstützung für die $P O$; es spiegelte eher den Wunsch wider, ersterer die Macht zu entziehen, als sie letzterer zu überantworten. Die Hauptmotivation war die Erwartung, dass sich der Regierungsstil ändern würde und weniger Erwartungen hinsichtlich bestimmter politischer Entscheidungen und Vorhaben. Dies wurde durch die erklärte "Offensive der Nächstenliebe« von Donald Tusk und seiner $P O$ genährt, die einen Kontrast zur aggressiven Politik der Kaczyńskis darstellen soll, wobei gleichzeitig suggeriert wird, dass diese von Hass motiviert war. Die Spaltung der Gesellschaft endete für die Kaczyńskis mit einer Niederlage, denn sie brachten die gesellschaftliche Mehrheit gegen sich auf. Ausdruck dessen sind nicht nur die von PiS verlorenen Wahlen, sondern auch die niedrige Bewertung der Präsidentschaft von Lech Kaczyński nach dreijähriger Amtsausübung.

Die Beruhigung der hysterischen Atmosphäre in der Politik, die die Mehrheit der Bevölkerung erwartet hatte, trat tatsächlich ein. Daran hatte auch die geschickte Regierungsbildung der siegreichen PO mit der Polnischen Bauernpartei (Polskie Stronnictwo Ludowe - PSL) als Juniorpartner Anteil. Dieses Bündnis hebt sich deutlich von den ständigen Streitigkeiten ab, die zwischen der PiS und ihren Koalitionspartnern ausbrachen und schließlich zu den vorgezogenen Neuwahlen führten. Außerdem wurden auch stabile, friedliche und routinierte Beziehungen zu den Nachbarstaaten wiederhergestellt, darunter besonders zu Deutschland. Diese Art und Weise, Politik und Regierungsmacht auszuüben, erfährt gesellschaftlich eine große Unterstützung, was sich an Umfrageergebnissen zur Bewertung der $P O$ ablesen lässt. Danach übersteigt die Unterstützung für die $P O$ nach einem Jahr Regierung Tusk sogar noch die Zustimmung zur PO zur Zeit der Wahlen. Größtenteils ist dies allerdings ein Ausdruck der Angst vor einer Rückkehr der PiS an die Macht, deren Vertreter immer noch eine aggressive, streitsüchtige Haltung demonstrieren und Konflikte provozieren.

An dieser Stelle sei darauf hingewiesen, dass die verbreitete Ablehnung der Polen gegenüber einer Politik, die in Form eines Kampfes oder einer permanenten Revolution verwirklicht wird, nicht dem Stereotyp entspricht, das die Nation als aufbrausend, unverträglich und bereit zu Aufstand und Revolte charakterisiert. 


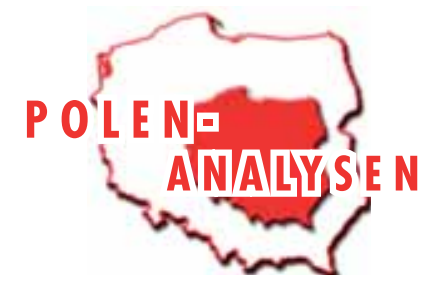

Dies deutet nicht nur auf die Gewöhnung und Verinnerlichung eines demokratischen Ethos hin, sondern auch auf die "Verbürgerlichung» der Gesellschaft, die immer stärker an einem stabilen privaten und öffentlichen Leben interessiert ist, das die bisherigen Errungenschaften zu erhalten und zu nutzen garantiert. Soziologische Untersuchungen zeigen seit Jahren, dass die Polen von der Politik die Fähigkeit zu Konsens und Kooperation erwarten. Die nicht geglückten Versuche, Konflikte zu entfachen und unterschiedliche gesellschaftliche Gruppen gegeneinander aufzubringen, zeigen den nennenswerten Unterschied zu Gesellschaften wie der spanischen oder italienischen, ganz zu schweigen von der ungarischen oder ukrainischen, die sich zu derlei Konflikten provozieren ließen und in immer tieferen politischen Spaltungen versinken.

\section{Vermeidung dringender Reformen}

Die von Tusk betriebene Politik der Beruhigung und Stabilisierung der Stimmung in der Gesellschaft hatte allerdings ihren Preis, nämlich die Vermeidung dringender und notwendiger Reformen, die gesellschaftliche Unruhen hervorrufen könnten. Die ersten Monate der Regierung waren also nicht nur eine Zeit der Stabilität und Ruhe, sondern auch der Stagnation, was immer häufiger und stärker von den unparteiischen Kommentatoren und sogar den Regierungs- oder $P O$-Sympathisanten bemängelt wird. Die »Offensive der Nächstenliebe«schloss eine Reformoffensive aus. Es gelang dabei jedoch nicht, Konflikten und Unstimmigkeiten vollständig auszuweichen, zumal sie von der aggressiven Antiregierungspolitik und -rhetorik der PiS hervorgerufen wurden sowie von Staatspräsident Lech Kaczyński, der die Rolle des Hauptgegners der Regierung spielt und sich bemüht, immer aktiver auf ihre Politik (besonders die Außenpolitik) einzuwirken bzw. sie zu sabotieren. Hinzu kommt die Finanz- und Wirtschaftskrise, die zwar nicht primär in Polen stattfindet, deren Auswirkungen jedoch unvermeidlich zu spüren sind.

Die neuralgischen Punkte der polnischen Reformpolitik sind typisch für zeitgenössische, entwickelte europäische Gesellschaften, und zwar sind es das Rentensystem, das Gesundheitswesen, das Bildungssystem und andere Bereiche der Gesellschaftspolitik. In fast allen diesen Fragen stellte die Regierung ihre Vorschläge dar, die erwartungsgemäß Proteste und Einspruch verschiedener gesellschaftlicher Gruppen hervorriefen. Die Politik des Dialogs, der Suche nach Kompromissen und der Erarbeitung von Vereinbarungen, die in der Person des Beraters und Beauftragten des Ministerpräsidenten, Michał Boni, personifiziert ist, zeitigte nur Teiler- folge. Die Gewerkschaften, die die stärkste und am besten organisierte Lobby verschiedener gesellschaftlicher Gruppen und Interessen sind, stellen sich gegen alle Projekte, die irgendein partikuläres Privileg oder Anrecht berühren; es handelt sich dabei auch oder gerade um solche, die noch aus der Zeit des Kommunismus stammen. Die Lage der Regierung verschlimmert die Tatsache, dass die liberale $P O$ keine besondere Beziehung zu den Gewerkschaftszentralen hat, wie es traditionell bei der Linken und der Rechten der Fall ist, so dass diese zumindest auf wohlwollende Neutralität der befreundeten Gewerkschaft zählen können. Die $P O$ hat sie alle gegen sich. Hinzu kommt, dass diese im Widerstand gegen die »liberalen" Reformen ihre Kräfte vereinen, indem die antikommunistische Solidarność und der postkommunistische Gewerkschaftsbund Gesamtpolnische Verständigung der Gewerkschaften (Ogólnopolskie Porozumienie Zwiazków Zawodowych-OPZZ) ihre alten Animositäten überwinden. Die gemäßigte Unterstützung, die Arbeitgebervertretungen der $P O$ und der Regierung zuteil werden lassen, sind nicht im Stande, die ablehnende Einstellung der Gewerkschaften gegenüber der Regierung auszugleichen.

\section{Probleme mit Gegnern und Partnern}

Die Regierung handelt in einer sie behindernden und ihr feindlich gesonnenen Umgebung ohne Verbündete. Die von der Regierungsverantwortung abgelöste PiS nahm eine Haltung kompromissloser Ablehnung ein, was zu erwarten war. Eine gewisse Überraschung bot aber die Politik der Linken, von der zumindest wohlwollende Neutralität gegenüber der Regierung erwartet worden war. Diese ist ja immerhin ein Antidotum gegen die Exzesse der PiS, welche auch vom linken Wähler und Establishment sehr kritisch beurteilt wurden. Jedoch machte sich nach dem schwachen Wahlergebnis, dass durch den Wechsel vieler Links-Wähler zur $P O$ verursacht worden war, die als einzig fähige Kraft, die PiS abzulösen beurteilt wurde, in der Demokratischen Linksallianz (Sojusz Lewicy Demokratycznej-SLD) Kritik breit und es wurde mit der bisherigen Führungsriege abgerechnet, die für die Wahlniederlage verantwortlich gemacht wurde. Neuer Vorsitzender ist der junge Dogmatiker postkommunistischer Provenienz Grzegorz Napieralski, der die Rückkehr zur linken, radikal antiliberalen - und demnach gegen die Regierung ausgerichteten - Orthodoxie forciert. Das bedeutet, dass die Regierung nicht auf die Stimmen der Linken zählen kann, wenn ihre Initiativen durch das Veto des Staatspräsidenten blockiert oder sabotiert werden, wovon Lech Kaczyński oft und gern Gebrauch macht 


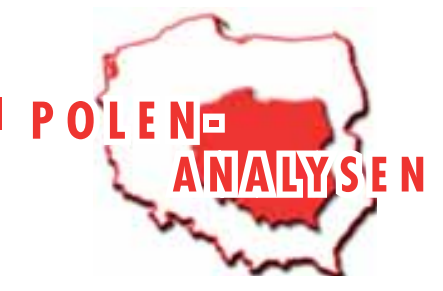

polen-analysen $43 / 08$

und womit er den Erwartungen und Wünschen seines Bruders Jarosław, des Vorsitzenden der stärksten Oppositionspartei PiS, entspricht. Die Regierung hat also alle Gewerkschaften gegen sich, die gesamte (linke und rechte) Opposition und den Präsidenten. Darüber hinaus hat die $P O$ ein Problem mit dem Koalitionspartner PSL, der sich als Partei der Landbevölkerung allen Reformversuchen für das übervölkerte und zurückgebliebene polnische Dorf sowie für den anachronistischen und ineffektiven Landwirtschaftssektor mit seiner archaischen Agrarstruktur entgegenstellt. Dies stellt ein wesentliches Problem dar, wenn die Regierung Reformen in der Gesellschaftspolitik durchführen will, von denen die Landbevölkerung ausgeklammert bleiben soll. Dass diese alle bisherigen Privilegien und Rechte behalten darf, soll aber dann vom Rest der Gesellschaft mitgetragen werden. Die von Politikern der PSL vorgeschlagenen Änderungen im verschwenderischen Rentensystem der Bauern erwiesen sich als nur kosmetische Korrektur einer Rentenpolitik, die jährlich Milliarden des Staatshaushalts verschlingt.

Der Druck auf die Regierung wird allerdings dadurch vermindert, dass die zahlreichen Gegner schwach sind und immer schwächer werden. Die PiS, unfähig, ihre aggressive Rhetorik und streitsüchtige Strategie abzulegen, hat einen konstant geringen Rückhalt in der Gesellschaft, weniger als zur Zeit der Wahlen 2007 und halb so viel wie die PO. Ähnlich schwach sind die Umfrageergebnisse für Staatspräsident Kaczyński und seine Chancen, wiedergewählt zu werden, sind gering. Es steigt also die Wahrscheinlichkeit seiner Niederlage gegen den derzeitigen Favoriten für das Amt des Staatspräsidenten in zwei Jahren, Donald Tusk, womit ein Hindernis für die Regierungstätigkeit der $P O$ ausgeräumt wäre. Die Linke mit ihrer neuen Führung müht sich nach wie vor am Rand ab und ihre gegen die Regierung eingestellte Strategie macht es ihr unmöglich, die Rolle des Schiedsrichters in Konflikten zwischen der Regierung und dem Staatspräsidenten zu spielen, wozu sie aber eigentlich ihre parlamentarische Position berechtigen würde.

Negativen Einfluss auf die Tätigkeit der Regierung hat auch die Finanz- und Wirtschaftskrise, die aus von ihr unabhängigen Gründen zutage trat. Dass es keine bedrohlichen und turbulenten Einbrüche und Ereignisse im polnischen Finanz- und Wirtschaftssystem gab (sieht man von dem Zusammenbruch der Börse ab, der mit dem globalen Trend einherging, sowie vom Kursverfall der heimischen Währung), weist auf die große Widerstandskraft gegen den Druck der negativen Prozesse hin, die in der globalen Wirtschaft und anderen Volkswirtschaften innerhalb der EU ablaufen. Die
Regierung reagiert adäquat, indem sie die Balance zwischen der notwendigen Wachsamkeit und der Vermeidung gesellschaftlicher Besorgnis durch zu heftige Reaktionen hält. Die Gesellschaft verhält sich überraschend ruhig und zeigt weder Hysterie noch Panik. Wenn nicht noch eine gewaltige, von außen hervorgerufene Erschütterung eintritt, hat die polnische Wirtschaft die Chance eines sanften Übergangs in eine Phase langsamerer Entwicklung und könnte eine Rezession umgehen.

\section{Bewährungsproben}

Dies hängt auch von der Politik der Polnischen Nationalbank ab, einer weiteren Institution, die der Regierung nicht wohl gesonnen ist und von einem wenig erfahrenen und wenig kompetenten Vorsitzenden geleitet wird. Er wird von Staatspräsident Kaczyński protegiert und ist eng mit diesem verbunden. In letzter Zeit sind jedoch immer mehr und stärkere Signale einer guten Zusammenarbeit zwischen der Bank und der Regierung zu beobachten. Einer der ausschlaggebenden Impulse war der von Ministerpräsident Tusk vorgestellte Plan, wonach Polen 2012 der Eurozone beitreten soll. Die Umsetzung dieses prestigereichen Projekts erfordert nicht nur die Koordination mit der Nationalbank (die Zurückhaltung demonstriert, und sei es nur aus dem Grund, dass die Einführung der europäischen Währung in Polen das Ende der Existenz der polnischen Nationalbank bedeuten würde), sondern auch eine verantwortungsbewusste makroökonomische Politik. Der aus Großbritannien reemigrierte Finanzminister Jacek Rostowski gilt als fähiger Experte, doch die erwartete wirtschaftliche Verlangsamung wird die Haushaltseinnahmen im nächsten Jahr verringern, so dass also eine Kürzung der Ausgaben oder eine Ausweitung des Haushaltsdefizits erforderlich sein wird. Ersteres würde die Proteste derjenigen gesellschaftlichen Gruppen hervorrufen, die von einer solchen Reduktion betroffen wären, letzteres dagegen würde die Erfüllung der Voraussetzungen in Frage stellen, die von einem Beitrittskandidaten verlangt werden, und möglicherweise den Beitrittsprozess selbst verlangsamen. Die Entscheidung wird nicht leicht werden und die Opposition wird sie sicherlich nicht erleichtern. Die PiS sabotiert bereits den Plan, den Euro 2012 einzuführen, wofür eine Verfassungsänderung notwendig ist (und zwar der Teile, die der Polnischen Nationalbank ihre Unabhängigkeit in der Geldpolitik garantieren und ihr die Ausgabe des sich im landesweiten Umlauf befindlichen Geldes übertragen).

Das zweite Prestigeprojekt, gleichen Namens und Zeitraums, ist die Organisation der Fußballeuropameisterschaft EURO 2012, die in Polen und der Ukraine 


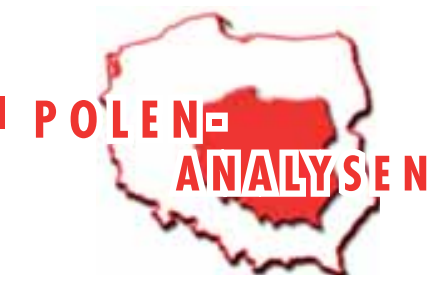

ausgetragen wird. Dafür ist es notwendig, bedeutenden Aufwand (finanzieller und organisatorischer Art) im Bereich Infrastruktur zu betreiben und viele Investitionen vorzunehmen, um den Standard zu verbessern. Der Stand der Vorbereitungen und die Fortschritte in diesem Bereich werden ein wichtiges Kriterium zur Beurteilung der Regierungstätigkeit sein. Zurzeit ist eine systematische Verbesserung und zunehmende Dynamik festzustellen; allerdings könnten ambitionierte Straßenbauprojekte (Autobahnen) unverwirklicht bleiben, was sich negativ auf das Ansehen der Regierung niederschlagen würde - insbesondere, da Donald Tusk seine Vorgänger scharf für ihre Saumseligkeit kritisiert hat und die Kompetenz im Bereich Autobahnbau in der öffentlichen Meinung zu einem der wichtigsten Kriterien für die Beurteilung der Regierung wurde.

Die größte Bewährungsprobe wird aber die Einführung von Renten- und Gesundheitsreformen sein. In der ersten Frage sind die Entscheidungen bereits getroffen, das entsprechende Gesetz (die eingeschränkte Möglichkeit des Eintritts in den vorzeitigen Ruhestand) wurde vom Parlament verabschiedet und auch die sich verstärkenden gesellschaftlichen Proteste können keine Rücknahme dieser Änderungen erzwingen. Auch der Staatspräsident hat nur begrenzte Möglichkeiten, es zu blockieren, denn wenn es nicht in Kraft träte, würden vom 1. Januar 2009 an alle bisherigen Rentenprivilegien ersatzlos erlöschen.

Die Reformen im Gesundheitssystem konzentrieren sich auf die kommerzielle Umstrukturierung medizinischer Einrichtungen, vor allem von Krankenhäusern, die unter der Schuldenlast zusammenzubrechen drohen, obwohl die Schulden häufig vom Staat gezahlt bzw. eingefroren wurden. Die Opposition und der Präsident sind dagegen und malen das Schreckensbild einer
Privatisierung des Gesundheitswesens. Dies entspricht jedoch nicht der Wahrheit, da die kommerzialisierten medizinischen Einrichtungen den Patienten kostenlose Dienstleistungen anbieten und dafür von den Krankenkassen bezahlt würden. Die Kommerzialisierung würde vielmehr der Transparenz der Verwaltung und Finanzkontrolle dienen. Die Regierung kann diesen Widerstand umgehen, indem sie eine angemessene außergesetzmäßige Transformation mit Hilfe der Selbstverwaltungen ermöglicht. Dies würde aber nicht das grundlegende Problem der öffentlichen Gesundheitsfürsorge lösen, das in der Regulierung des Zutritts zu kostenlosen und immer differenzierteren und komplizierteren - und dadurch auch immer kostspieligeren - Behandlungen besteht.

Zusammenfassend lässt sich feststellen, dass die Regierung von Ministerpräsident Tusk nach einer mehrmonatigen Phase des Stillstands in eine gesetzgebende und reformierende Offensive eingetreten ist, die allerdings auf den Widerstand einer voreingenommenen oder sogar feindlich eingestellten Umgebung stößt. Die Art und Weise, wie die Regierung damit umgeht, wird über die Umsetzung der dargestellten ambitionierten Pläne mitentscheiden. Die globale Finanz- und Wirtschaftskrise schafft außerdem weitere Gefahren und Probleme, die es der Regierung erschweren können, erfolgreich zu sein. Es bleibt abzuwarten, ob und in welchem Maß die öffentliche Meinung die Regierung für sich daraus eventuell ergebende Misserfolge verantwortlich macht. Bisher unterstützt die Öffentlichkeit die Regierung und vertraut ihr, aber es gibt Anzeichen, dass der Vertrauenskredit deutlich schmilzt, unabhängig von den allgemeinen Schwierigkeiten auf dem Kreditmarkt.

Übersetzung aus dem Polnischen: Silke Plate

\section{Über den Autor:}

Janusz A. Majcherek ist Professor am Institut für Philosophie und Soziologie an der Pädagogischen Universität Krakau. Seine Analysen und Kommentare zur polnischen Politik, Gesellschaft und Wirtschaft werden v. a. in der Tageszeitung »Gazeta Wyborcza« und der katholischen Wochenzeitung »Tygodnik Powszechny« sowie in Fernseh- und Radiosendungen veröffentlicht. 


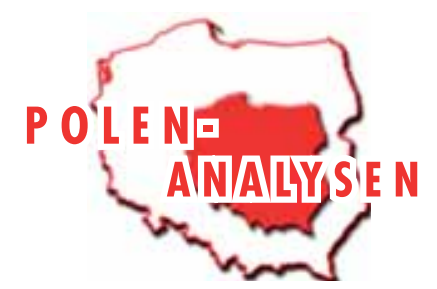

\section{Tabellen und Grafiken}

\section{Die Regierung Tusk im Spiegel von Meinungsumfragen}

Wie beurteilen Sie die Tätigkeit der Regierung von Donald Tusk?

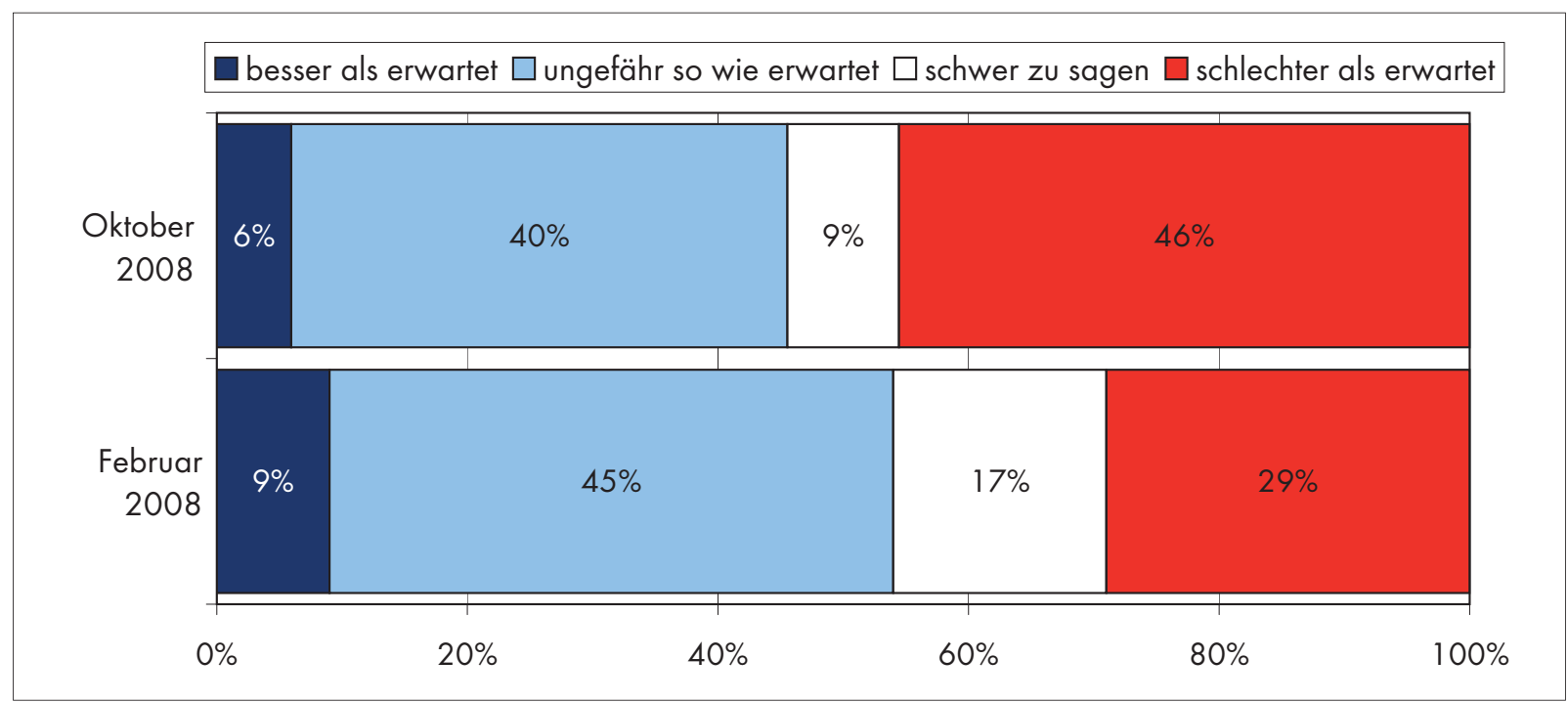

Quelle: CBOS BS 170/2008: Sukcesy i zaniechania rzadu Donalda Tuska [Erfolge und Versäumnisse der Regierung von Donald Tusk], Warszawa 11/2008, www.cbos.pl

Die Regierung von Donald Tusk...

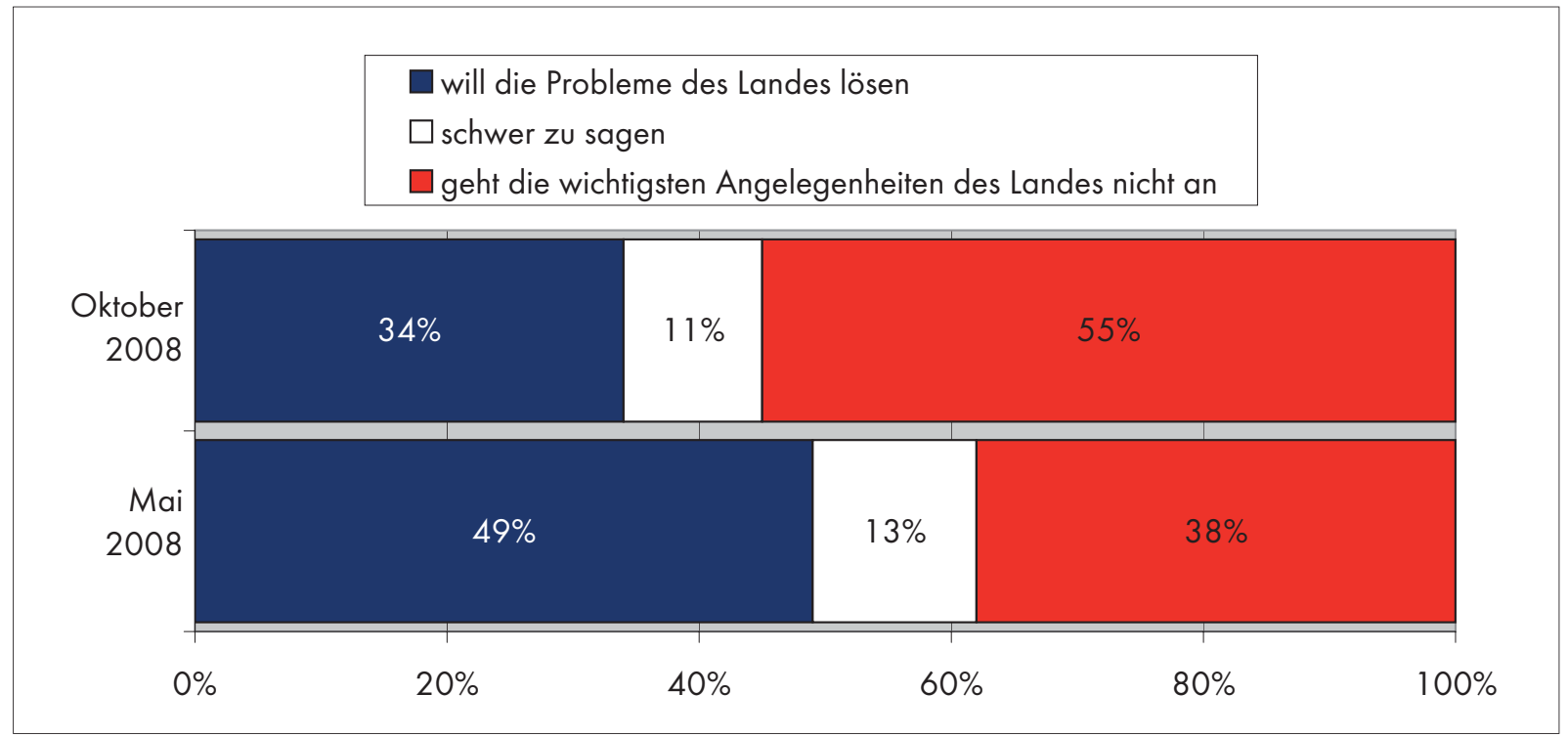

Quelle: CBOS BS 170/2008: Sukcesy i zaniechania rzadu Donalda Tuska [Erfolge und Versäumnisse der Regierung von Donald Tusk], Warszawa 11/2008, ww w.cbos.pl 


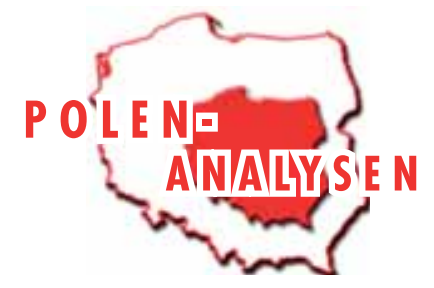

Die Regierung von Donald Tusk...

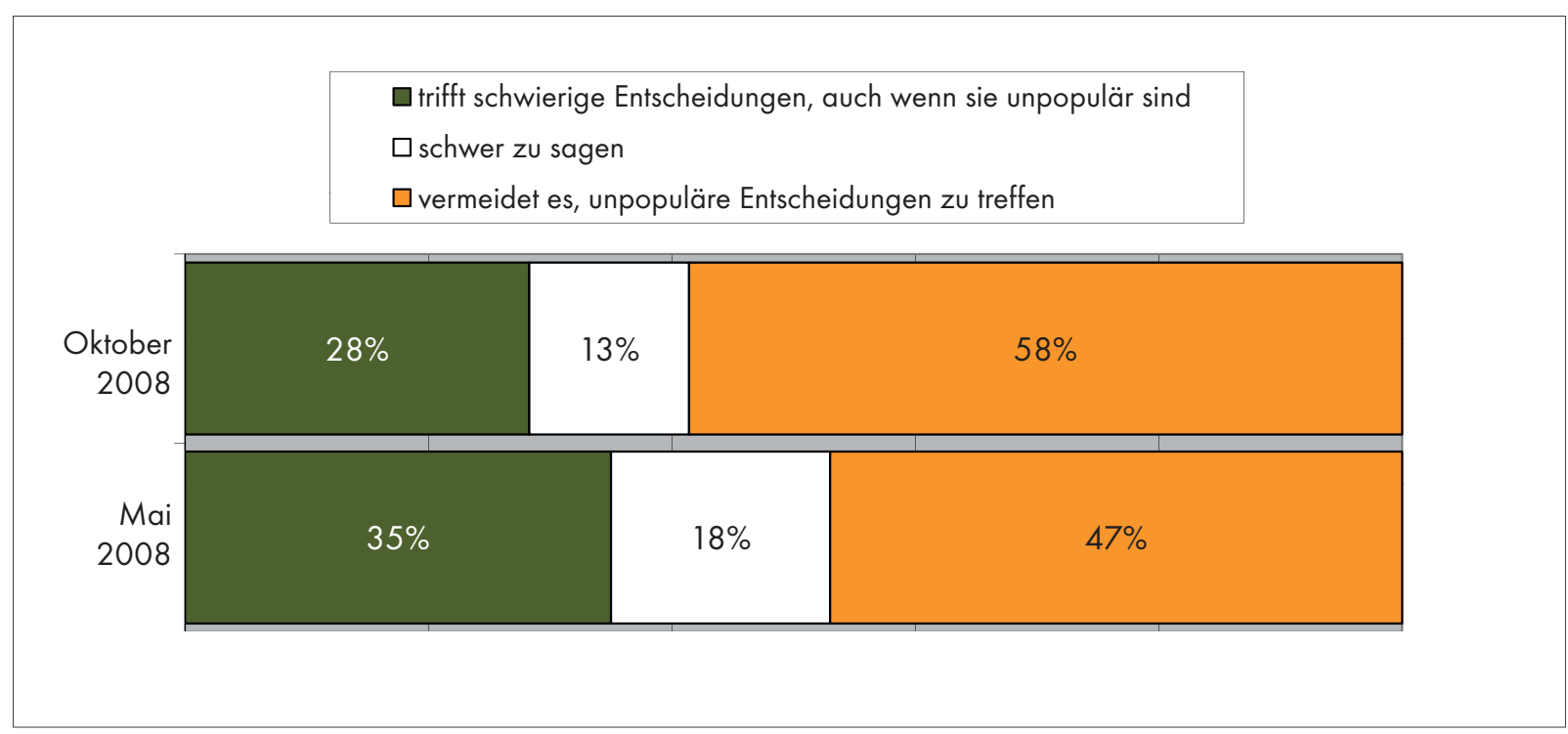

Quelle: CBOS BS 170/2008: Sukcesy i zaniechania rzadu Donalda Tuska [Erfolge und Versäumnisse der Regierung von Donald Tusk], Warszawa 11/2008, ww w.cbos.pl

Wie beurteilen Sie die Zusammenarbeit in der Regierungskoalition aus PO und PSL?*

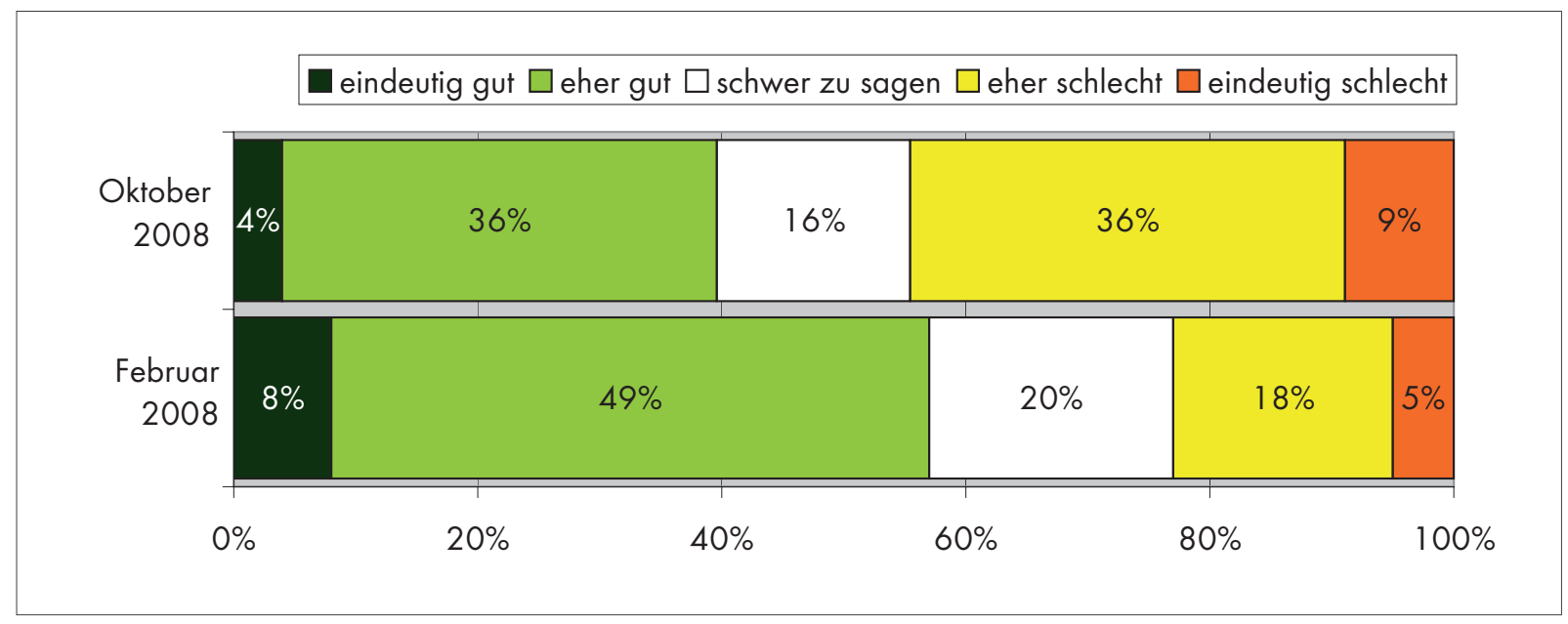

*PO - Platforma Obywatelska (Bürgerplattform), PSL - Polskie Stronnictwo Ludowe (Polnische Bauernpartei)

Quelle: CBOS BS 170/2008: Sukcesy i zaniechania rzadu Donalda Tuska [Erfolge und Versäumnisse der Regierung von Donald Tusk], Warszawa 11/2008, www.cbos.pl 


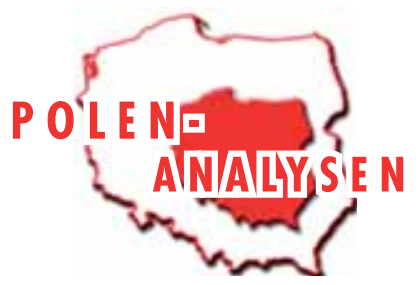

Wie beurteilen Sie die Zusammenarbeit zwischen der Regierung von Ministerpräsident Donald Tusk und Staatspräsident Lech Kaczyński? (\%)

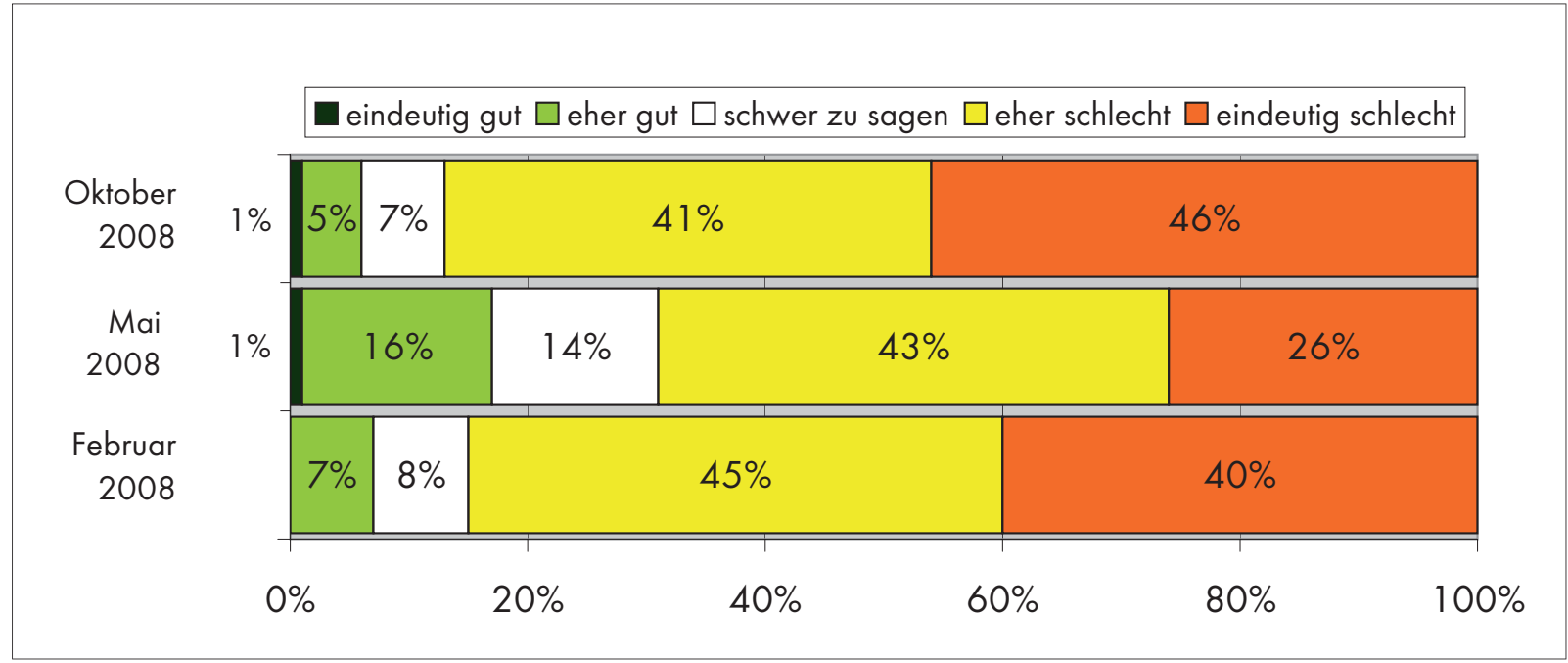

Quelle: CBOS BS 170/2008: Sukcesy i zaniechania rzadu Donalda Tuska [Erfolge und Versäumnisse der Regierung von Donald Tusk], Warszawa 11/2008, www.cbos.pl

Ermöglicht die gegenwärtige Regierung eine Verbesserung der wirtschaftlichen Situation?

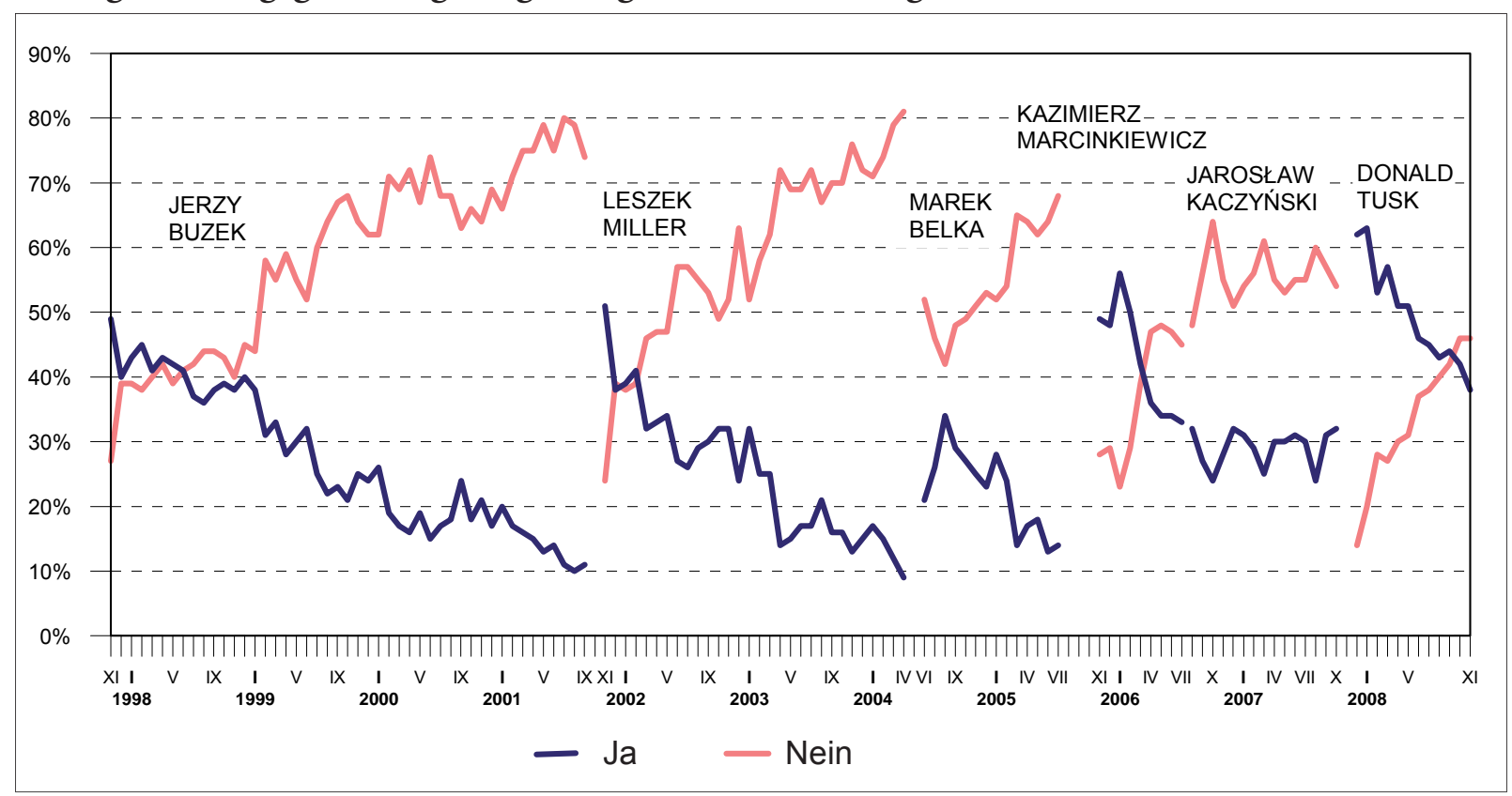

(Anm.: Die Antwort »schwer zu sagen « wurde nicht dargestellt.)

Quelle: CBOS BS 171/2008: Stosunek do rządu Donalda Tuska w listopadzie [Das Verhältnis zur Regierung von Donald Tusk im November], Warszawa 11/2008, w w w.cbos.pl 


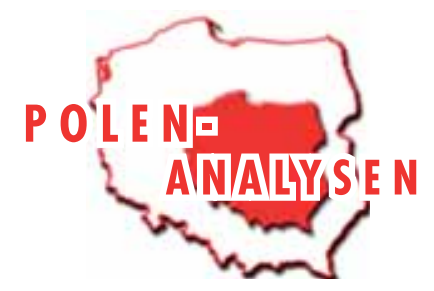

Beurteilung der Regierungstätigkeit in verschiedenen Bereichen (\%)

\begin{tabular}{|c|c|c|c|c|c|c|c|c|}
\hline & Mai 2002 & Feb. 2003 & März 2004 & Okt. 2005 & Feb. 2007 & Sept. 2007 & Mai 2008 & Okt. 2008 \\
\hline & \multicolumn{3}{|c|}{ Leszek Miller } & $\begin{array}{c}\text { Marek } \\
\text { Belka }\end{array}$ & \multicolumn{2}{|c|}{ Jarosław Kaczyński* } & \multicolumn{2}{|c|}{ Donald Tusk } \\
\hline \multicolumn{9}{|l|}{ Wirtschaft } \\
\hline gut & 13 & 9 & 4 & 12 & 20 & 30 & 34 & 30 \\
\hline ausreichend & 51 & 51 & 37 & 49 & 48 & 42 & 45 & 49 \\
\hline ungenügend & 24 & 30 & 51 & 23 & 21 & 21 & 10 & 13 \\
\hline schwer zu sagen & 13 & 9 & 8 & 16 & 11 & 6 & 11 & 9 \\
\hline \multicolumn{9}{|l|}{ Staatsfübrung } \\
\hline gut & 28 & 20 & 6 & 14 & 21 & 22 & 43 & 35 \\
\hline ausreichend & 45 & 45 & 38 & 46 & 40 & 37 & 38 & 43 \\
\hline ungenügend & 16 & 21 & 48 & 23 & 30 & 36 & 10 & 13 \\
\hline schwer zu sagen & 12 & 14 & 8 & 17 & 9 & 5 & 9 & 9 \\
\hline \multicolumn{9}{|c|}{ Bewirtschaftung öffentlicher Gelder } \\
\hline gut & 11 & 5 & 2 & 9 & 12 & 18 & 27 & 23 \\
\hline ausreichend & 32 & 30 & 18 & 31 & 34 & 35 & 36 & 40 \\
\hline ungenügend & 36 & 47 & 68 & 39 & 39 & 34 & 15 & 22 \\
\hline schwer zu sagen & 21 & 18 & 12 & 21 & 15 & 14 & 22 & 15 \\
\hline \multicolumn{9}{|l|}{ Außenpolitik } \\
\hline gut & 37 & 34 & 21 & 23 & 16 & 21 & 50 & 41 \\
\hline ausreichend & 32 & 33 & 39 & 38 & 32 & 29 & 26 & 34 \\
\hline ungenügend & 12 & 14 & 21 & 15 & 35 & 38 & 9 & 10 \\
\hline schwer zu sagen & 19 & 19 & 19 & 23 & 17 & 13 & 15 & 16 \\
\hline \multicolumn{9}{|c|}{ Landwirtschaftspolitik } \\
\hline gut & 9 & 6 & 3 & 14 & 12 & 20 & 22 & 18 \\
\hline ausreichend & 29 & 32 & 26 & 37 & 38 & 37 & 32 & 38 \\
\hline ungenügend & 43 & 45 & 54 & 28 & 32 & 27 & 16 & 24 \\
\hline schwer zu sagen & 19 & 17 & 17 & 22 & 18 & 16 & 30 & 20 \\
\hline \multicolumn{9}{|c|}{ Fürsorge für diejenigen, die am meisten Hilfe brauchen } \\
\hline gut & 6 & 5 & 2 & 7 & 14 & 16 & 18 & 16 \\
\hline ausreichend & 27 & 29 & 18 & 26 & 30 & 32 & 40 & 40 \\
\hline ungenügend & 55 & 57 & 72 & 52 & 46 & 44 & 26 & 33 \\
\hline schwer zu sagen & 12 & 9 & 7 & 15 & 10 & 8 & 16 & 12 \\
\hline \multicolumn{9}{|c|}{ Sicherheit der Bürger } \\
\hline gut & 19 & 17 & 9 & 11 & 40 & 40 & 34 & 29 \\
\hline ausreichend & 40 & 39 & 34 & 33 & 36 & 34 & 35 & 39 \\
\hline ungenügend & 31 & 37 & 48 & 41 & 17 & 19 & 18 & 22 \\
\hline schwer zu sagen & 10 & 7 & 9 & 15 & 7 & 7 & 13 & 10 \\
\hline
\end{tabular}




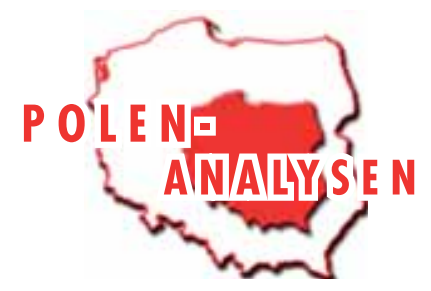

Beurteilung der Regierungstätigkeit in verschiedenen Bereichen (\%) (Fortsetzung)

\begin{tabular}{|c|c|c|c|c|c|c|c|c|}
\hline & Mai 2002 & Feb. 2003 & März 2004 & Okt. 2005 & Feb. 2007 & Sept. 2007 & Mai 2008 & Okt. 2008 \\
\hline & \multicolumn{3}{|c|}{ Leszek Miller } & $\begin{array}{c}\text { Marek } \\
\text { Belka }\end{array}$ & \multicolumn{2}{|c|}{ Jarosław Kaczyński* } & \multicolumn{2}{|c|}{ Donald Tusk } \\
\hline \multicolumn{9}{|c|}{ Schulwesen, Bildung } \\
\hline gut & 19 & 17 & 11 & 14 & 19 & 20 & 30 & 27 \\
\hline ausreichend & 41 & 42 & 40 & 36 & 34 & 35 & 40 & 41 \\
\hline ungenügend & 22 & 26 & 35 & 29 & 34 & 36 & 14 & 19 \\
\hline schwer zu sagen & 18 & 15 & 14 & 21 & 12 & 9 & 16 & 13 \\
\hline \multicolumn{9}{|l|}{ Gesundheitswesen } \\
\hline gut & 11 & 7 & 4 & 7 & 11 & 13 & 16 & 14 \\
\hline ausreichend & 32 & 26 & 18 & 22 & 26 & 32 & 36 & 37 \\
\hline ungenügend & 49 & 61 & 73 & 60 & 58 & 50 & 41 & 42 \\
\hline schwer zu sagen & 8 & 6 & 5 & 11 & 5 & 5 & 7 & 7 \\
\hline \multicolumn{9}{|c|}{ Informationspolitik } \\
\hline gut & 26 & 21 & 10 & 13 & 23 & 23 & 37 & 27 \\
\hline ausreichend & 40 & 40 & 35 & 37 & 39 & 35 & 34 & 41 \\
\hline ungenügend & 19 & 25 & 41 & 28 & 27 & 30 & 15 & 18 \\
\hline schwer zu sagen & 14 & 14 & 14 & 22 & 12 & 11 & 14 & 14 \\
\hline \multicolumn{9}{|c|}{ Bekämpfung der Korruption } \\
\hline gut & - & - & - & - & 35 & 40 & 28 & 25 \\
\hline ausreichend & - & - & - & - & 31 & 30 & 32 & 36 \\
\hline ungenügend & - & - & - & - & 24 & 20 & 21 & 26 \\
\hline schwer zu sagen & - & - & - & - & 10 & 9 & 19 & 14 \\
\hline \multicolumn{9}{|c|}{ Straßen- und Autobabnbau } \\
\hline gut & - & - & - & - & - & - & - & 19 \\
\hline ausreichend & - & - & - & - & - & - & - & 29 \\
\hline ungenügend & - & - & - & - & - & - & - & 45 \\
\hline schwer zu sagen & - & - & - & - & - & - & - & 7 \\
\hline \multicolumn{9}{|c|}{ Vorbereitungen der Fußballeuropameisterschaft EURO 2012} \\
\hline gut & - & - & - & - & - & - & - & 10 \\
\hline ausreichend & - & - & - & - & - & - & - & 29 \\
\hline ungenügend & - & - & - & - & - & - & - & 44 \\
\hline schwer zu sagen & - & - & - & - & - & - & - & 17 \\
\hline
\end{tabular}

* Anm.: Zur Amtsführung von Kazimierz Marcinkiewicz (31.10.2005 - 14.07.2006) wurden keine entsprechenden Daten erhoben. Quelle: CBOS BS 170/2008: Sukcesy i zaniechania rzadu Donalda Tuska [Erfolge und Versäumnisse der Regierung von Donald Tusk], Warszawa 11/2008, www.cbos.pl 


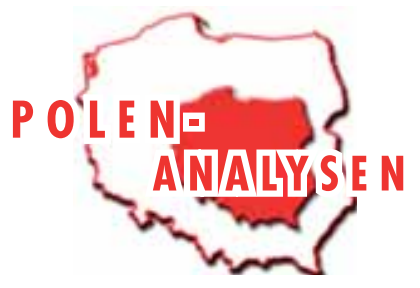

Wen würden Sie wählen, wenn am nächsten Sonntag Sejm- und Senatswahlen wären? (2008, \%)

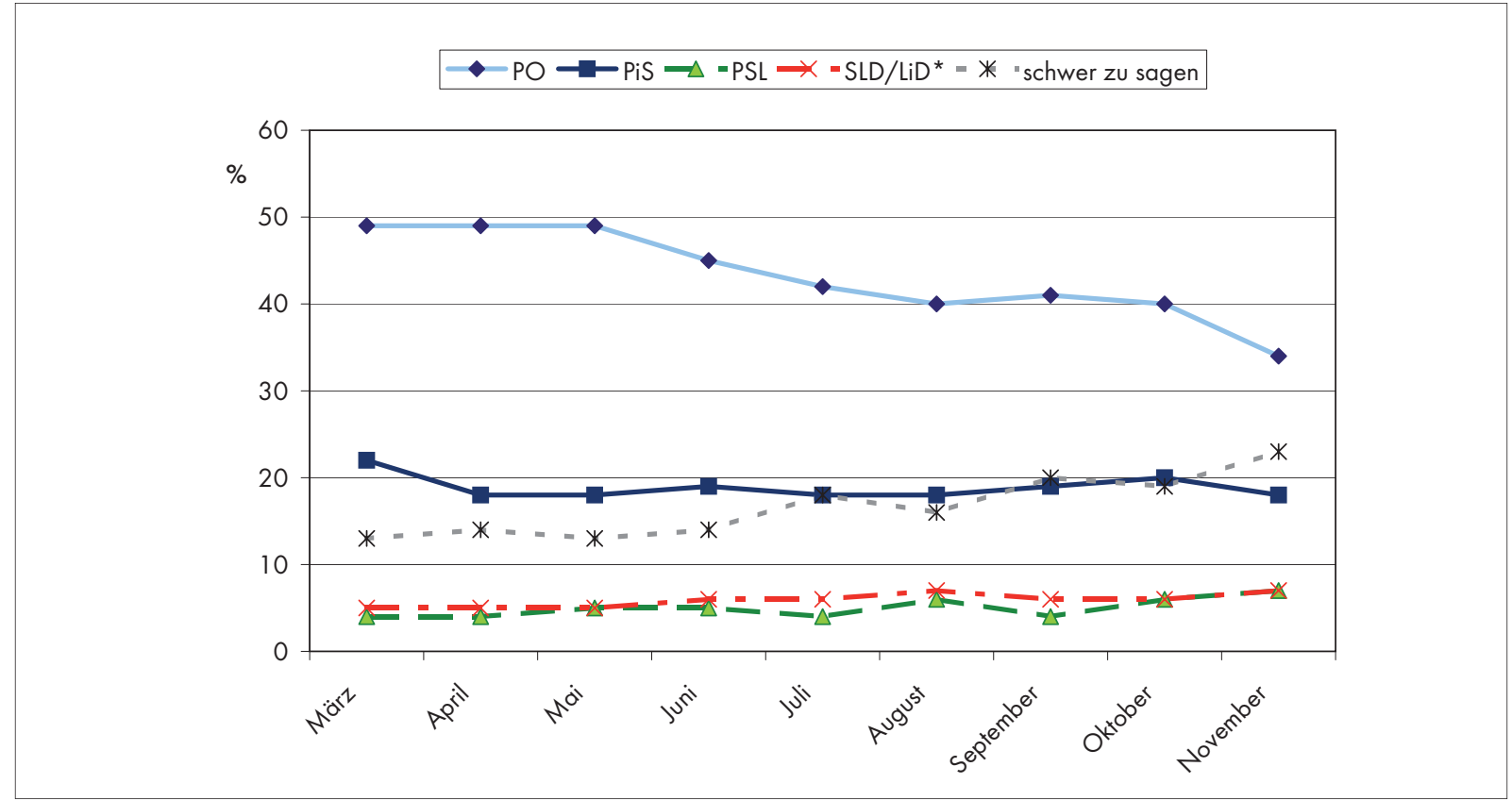

\begin{tabular}{|l|c|c|c|c|c|c|c|c|c|}
\hline & März 2008 & April 2008 & Mai 2008 & Juni 2008 & Juli 2008 & Aug. 2008 & Sept. 2008 & Okt. 2008 & Nov. 2008 \\
\hline PO & 49 & 49 & 49 & 45 & 42 & 40 & 41 & 40 & 34 \\
PiS & 22 & 18 & 18 & 19 & 18 & 18 & 19 & 20 & 18 \\
PSL & 4 & 4 & 5 & 5 & 4 & 6 & 4 & 6 & 7 \\
SLD/LiD* & 5 & 5 & 5 & 6 & 6 & 7 & 6 & 6 & 7 \\
LPR & 2 & 2 & 1 & 1 & 2 & 2 & 1 & 2 & 2 \\
Partia Kobiet & 2 & 2 & 2 & 2 & 2 & 2 & 2 & 1 & 2 \\
KPEiR & 2 & 1 & 1 & 2 & 1 & 3 & 2 & 1 & 1 \\
PD & - & 0 & 1 & 0 & 1 & 2 & 1 & 0 & 1 \\
PPP & 1 & 1 & 1 & 0 & 1 & 1 & 0 & 0 & 1 \\
Samoobrona & 0 & 1 & 1 & 2 & 2 & 1 & 1 & 1 & 1 \\
SdPl & - & 1 & 1 & 1 & 0 & 1 & 1 & 0 & 1 \\
\hline UPR & 0 & 1 & 0 & 1 & 1 & 1 & 0 & 1 & 1 \\
andere & 1 & 0 & 0 & 1 & 0 & 0 & 1 & 2 & 1 \\
\hline schwer zu & 13 & 14 & 13 & 14 & 18 & 16 & 20 & 19 & 23 \\
sagen & & & & & & & & & 1 \\
\hline
\end{tabular}

Anm.: Es werden nur die Parteien aufgelistet, die von mindestens $0,5 \%$ der Wähler gewählt würden.

* Koalition aus SLD, SdPl, PD und UP (Unia Pracy (Arbeitsunion)), 2008 aufgelöst

PO - Platforma Obywatelska (Bürgerplattform)

PiS - Prawo i Sprawiedliwość (Recht und Gerechtigkeit)

PSL - Polskie Stronnictwo Ludowe (Polnische Bauernpartei)

SLD - Sojusz Lewicy Demokratycznej (Demokratische Linksallianz)

LiD - Lewica i Demokraci (Linke und Demokraten)

LPR - Liga Polskich Rodzin (Liga der Polnischen Familien)

Partia Kobiet (Frauenpartei)

KPEiR - Krajowa Partia Emerytów i Rencistów (Landespartei der Pensionäre und Rentner)

PD - Partia Demokratyczna (Demokratische Partei)

PPP - Polska Partia Pracy (Polnische Arbeitspartei)

Samoobrona (Selbstverteidigung)

SdPl-Socjaldemokracja Polska (Polnische Sozialdemokratie)

UPR - Unia Polityki Realnej (Union für Realpolitik)

Quelle: CBOS BS/172/2008: Preferencje partyjne w listopadzie [Parteipräferenzen im November], Warszawa 11/2008, www.cbos.pl 


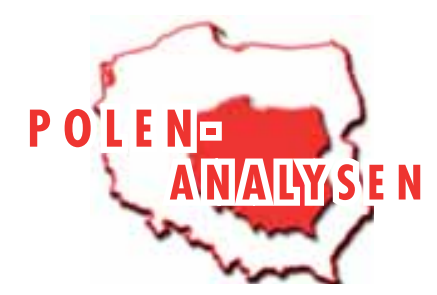

Chronik

\section{Vom 18. November bis zum 01. Dezember 2008}

\begin{tabular}{|c|c|}
\hline 18.11.2008 & $\begin{array}{l}\text { Verteidigungsminister Bogdan Klich kündigt an, dass bis Ende nächsten Jahres eine Batterie Patriot-Raketen in } \\
\text { Polen stationiert werde. Die Verhandlungen darüber sowie über die Stationierung einer amerikanischen Garnison } \\
\text { würden planmäßig verlaufen. }\end{array}$ \\
\hline 18.11.2008. & $\begin{array}{l}\text { In seiner Rede vor dem Nordatlantikrat in Washington unterstreicht Außenminister Radosław Sikorski, die Regierung } \\
\text { von Ministerpräsident Donald Tusk wolle keine Konfrontation mit Russland und keine Rückkehr zum Kalten } \\
\text { Krieg, jedoch dürfe nach der militärischen Intervention Russlands in Georgien und der Ankündigung des russischen } \\
\text { Staatspräsidenten Dimitrij Medwedjew nach der Wahl Barack Obamas zum US-Präsidenten, Russland werde in der } \\
\text { Exklave Kaliningrad als Reaktion auf den vereinbarten amerikanisch-polnischen Raketenabwehrschild Kurzstre- } \\
\text { ckenraketen stationieren, nicht so getan werden, als wäre in den Beziehungen zwischen dem Westen und Russland } \\
\text { nichts vorgefallen. Sikorski unterstreicht, Russland wolle, dass sich die USA aus Europa zurückzögen. Medwedjews } \\
\text { Doktrin fasst er folgendermaßen zusammen: Russland werde seine Landsleute und Infrastruktur im Ausland ver- } \\
\text { teidigen, wenn nötig auch mit Gewalt. Die NATO dürfe nun nicht ihr Versprechen einer NATO-Partnerschaft } \\
\text { gegenüber Georgien und der Ukraine zurückziehen. Sollte Russland sein Vorgehen gegenüber Georgien auch auf } \\
\text { die Ukraine anwenden, werde dies eine europäische Krise großen Ausmaßes auslösen und müsse zu einer Reaktion } \\
\text { des transatlantischen Bündnisses führen. }\end{array}$ \\
\hline 20.11 .2008 & $\begin{array}{l}\text { Nach einem Jahr Amtszeit zieht Ministerpräsident Donald Tusk vor dem Sejm eine Bilanz der Arbeit der Regierungs- } \\
\text { koalition aus Bürgerplattform (Platforma Obywatelska - PO) und Polnischer Bauernpartei (Polskie Stronnictwo } \\
\text { Ludowe - PSL). Außenpolitisch hebt Tusk die Entspannung des polnisch-deutschen Dialogs hervor und die } \\
\text { Stärkung der Position Polens in der Europäischen Union. Der polnisch-russische Dialog sei allerdings durch den } \\
\text { russisch-georgischen Konflikt gestört worden. Tusk appelliert an Oppositionsführer Jarosław Kaczyński, Staats- } \\
\text { präsident Lech Kaczyński zur Unterschrift des EU-Reformvertrags zwecks Ratifizierung zu bewegen, den beide } \\
\text { gemeinsam ausgehandelt hätten. }\end{array}$ \\
\hline 22.11 .2008 & $\begin{array}{l}\text { Auf dem Parteitag der Demokratischen Linksallianz (Sojusz Lewicy Demokratycznej-SLD) in Warschau bekräftigt } \\
\text { der Parteivorsitzende Grzegorz Napieralski, dass die SLD auf die lokalen Selbstverwaltungen setze. In ihnen sehe er } \\
\text { eine Chance für die Linke. Außerdem appelliert er für eine Geschichtsschreibung, die die Geschichte nicht entstelle, } \\
\text { die nicht nur Lech Wałęsa würdige, sondern auch Wojciech Jaruzelski, nicht nur Tadeusz Mazowiecki, sondern } \\
\text { auch Mieczysław Rakowski, sowohl Jacek Kuroń als auch Aleksander Kwaśniewski. }\end{array}$ \\
\hline 23.11 .2008 & $\begin{array}{l}\text { Auf der Fahrt zu einem Flüchtlingslager an der georgisch-südossetischen Grenze fallen in der Nähe des Konvois, } \\
\text { der die Staatspräsidenten von Georgien und Polen, Michail Saakaschwili und Lech Kaczyński, fährt, Schüsse. Das } \\
\text { Fahrtziel war der erste Programmpunkt des Besuchs von Kaczyński anlässlich des 5. Jahrestages der Rosenrevolution } \\
\text { in Georgien. Unklar ist, wer die Schüsse abgegeben hat und ob sie auf den Konvoi gerichtet waren. Kaczyński } \\
\text { vermutet russische Täter. }\end{array}$ \\
\hline 24.11 .2008 & $\begin{array}{l}\text { Außenminister Radosław Sikorski teilt mit, sein Ressort habe sich mit der Forderung nach vollständiger Aufklärung } \\
\text { des Vorfalls am Vortag in Georgien an die georgische Regierung gewandt sowie mit einer diesbezüglichen Nach- } \\
\text { frage an Russland. }\end{array}$ \\
\hline 25.11 .2008 & $\begin{array}{l}\text { Nach einem Urteil des Verfassungsgerichts haben Journalisten und Personen, die nicht Wissenschaftler sind, wei- } \\
\text { terhin keinen freien Zugang zu den Akten des Instituts des Nationalen Gedenkens (Instytut Pamięci Narodowej } \\
\text { - IPN). Für die Akteneinsicht bedarf es einer Empfehlung des redaktionellen bzw. wissenschaftlichen Vorgesetzten. } \\
\text { Das Gericht lehnt damit eine Klage des Bürgerrechtsbeauftragten Janusz Kochanowski ab und stimmt mit der } \\
\text { Staatsanwaltschaft und der Helsinki-Stiftung für Menschenrechte überein, dass es sich um sensible Informationen } \\
\text { handele, die nicht allgemein zugänglich sein sollten. }\end{array}$ \\
\hline 26.11 .2008 & $\begin{array}{l}\text { Staatspräsident Lech Kaczyński legt gegen drei von sechs Gesetzen des Gesundheitsreformpakets der Regierung ein } \\
\text { Veto ein. Kaczyński begründet sein Veto damit, dass er sein Wahlversprechen einlöse, die Privatisierung des Gesund- } \\
\text { heitswesens nicht zu unterstützen, und er außerdem keine Einwilligung zur Durchführung eines Referendums zur } \\
\text { Gesundheitsreform erhalten habe. Um das Veto aufzuheben, bedarf es einer 3/5-Mehrheit im Sejm, über die die } \\
\text { Regierungskoalition aus Bürgerplattform (Platforma Obywatelska - PO) und Polnischer Bauernpartei (Polskie } \\
\text { Stronnictwo Ludowe - PSL) nicht verfügt. Ministerpräsident Donald Tusk stellt in Aussicht, dass die Regierung } \\
\text { gemeinsam mit den lokalen Selbstverwaltungen die wirtschaftliche Umstrukturierung der Krankenhäuser durch- } \\
\text { führen werde. }\end{array}$ \\
\hline
\end{tabular}




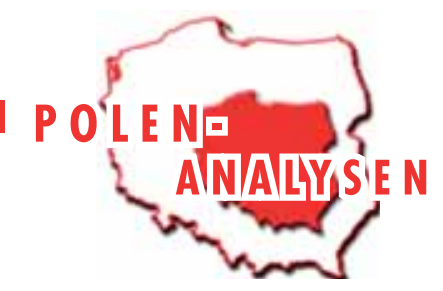

\begin{tabular}{|c|c|}
\hline 26.11.2008 & $\begin{array}{l}\text { Aufgrund des Besuches des Dalai Lama in Danzig aus Anlass des 25. Jahrestages der Verleihung des Friedens- } \\
\text { nobelpreises an Lech Wałesa Anfang Dezember sagt die chinesische Regierung den EU-China-Wirtschaftsgipfel } \\
\text { für Mitte Dezember ab. }\end{array}$ \\
\hline 27.11.2008 & $\begin{array}{l}\text { Die Regierung veröffentlicht einen Bericht zu dem Vorfall in der vergangenen Woche in Georgien, als in der Nähe } \\
\text { des Konvois, mit dem Staatspräsident Lech Kaczyński und sein georgischer Amtskollege Michail Saakaschwili } \\
\text { fuhren, Schüsse fielen. Demnach habe es sich weder um eine georgische Provokation noch um einen Attentatsver- } \\
\text { such gehandelt. Hinsichtlich der Sicherheit habe es organisatorische Unzulänglichkeiten gegeben. Der Chef des } \\
\text { Sicherheitsdienstes des Präsidenten wird daher vorläufig suspendiert. }\end{array}$ \\
\hline 29.11 .2008 & $\begin{array}{l}\text { Rafał Dutkiewicz, Vorsitzender der Bürgerbewegung Polen XXI (Ruch Obywatelski Polska XXI) und Stadtprä- } \\
\text { sident von Breslau, spricht sich auf der ersten programmatischen Konferenz der Bewegung in Warschau für die } \\
\text { Direktwahl von Woiwodschaftsmarschällen und Starosten sowie für die Umstrukturierung der Wahlkreise aus. Die } \\
\text { Bildung und Entwicklung der Selbstverwaltungen stellt er als große Leistung des vergangenen Jahrhunderts heraus. } \\
\text { Des Weiteren setze sich die Bewegung für eine starke Staatspräsidentschaft und eine leistungsstarke Regierung ein. } \\
\text { Kazimierz Ujazdowski, ehemaliger Kulturminister und ehemaliges Mitglied von Recht und Gerechtigkeit (Prawo } \\
\text { i Sprawiedliwość - PiS), kritisiert die gegenwärtige Politik in Polen als passiv und anachronistisch. Polen brauche } \\
\text { verantwortungsvolle Politiker, an denen es in der Politik derzeit mangele. Rafał Dutkiewicz sei eine solche Per- } \\
\text { sönlichkeit. An der Konferenz nahmen weitere Politiker aus der regionalen und überregionalen Politik teil. }\end{array}$ \\
\hline 30.11 .2008 & $\begin{array}{l}\text { Ministerpräsident Donald Tusk stellt ein Konjunkturprogramm der Regierung in Höhe von } 24 \text { Mrd. Euro als } \\
\text { Antwort auf die globale Finanzkrise vor. Es sieht Bankbürgschaften, Kredite für kleine und mittlere Unternehmen, } \\
\text { Investitionen in erneuerbare Energien und die beschleunigte Ausgabe von EU-Strukturmitteln vor. Die Prognose } \\
\text { des Wirtschaftswachstums wird von 4,8 auf 3,7 \% korrigiert. Ein Anstieg der Arbeitslosigkeit wird nicht voraus- } \\
\text { gesagt. }\end{array}$ \\
\hline 1.12 .2008 & $\begin{array}{l}\text { In Posen beginnt der Klimagipfel der Vereinten Nationen, auf dem u. a. eine Vereinbarung über die Reduzierung } \\
\text { von Treibhausgasen angestrebt wird, die im Dezember } 2009 \text { in Kopenhagen unterschrieben werden soll. In seiner } \\
\text { Eröffnungsrede unterstreicht Ministerpräsident Donald Tusk, dass der Umweltschutz unabhängig von Finanzkrisen } \\
\text { und wirtschaftlicher Konjunktur betrieben werden müsse. }\end{array}$ \\
\hline
\end{tabular}




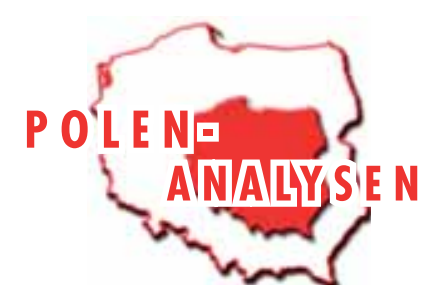

Über die Polen-Analysen

Die Polen-Analysen erscheinen zweimal monatlich als E-Mail-Dienst. Sie werden gemeinsam vom Deutschen PolenInstitut Darmstadt, der Bremer Forschungsstelle Osteuropa und der Deutschen Gesellschaft für Osteuropakunde herausgegeben. Die Herausgeber danken der BSH Bosch und Siemens Hausgeräte GmbH München und dem Stifterverband für die Deutsche Wissenschaft für ihre Unterstützung.

Ein Archiv der Polen-Analysen finden Sie im Internet unter w w w.laender-a na lysen.de/ polen

Kostenloses Abonnement unter http :// w w w.deutsches-polen-institut.de/ N ew sletter/ subscribe.php

\section{Deutsches Polen-Institut Darmstadt}

Das Deutsche Polen-Institut Darmstadt (DPI) ist ein Forschungs-, Informations-, und Veranstaltungszentrum für polnische Kultur, Geschichte, Politik, Gesellschaft und die deutsch-polnischen Beziehungen, die sich im Kontext der europäischen Integration entwickeln. Das seit März 1980 aktive und bis 1997 von Gründungsdirektor Karl Dedecius geleitete Institut ist eine Gemeinschaftsgründung der Stadt Darmstadt, der Länder Hessen und Rheinland-Pfalz sowie des Bundes. Seit 1987 ist die Trägerschaft auf die Kultusminister der Länder ausgedehnt. Einen wesentlichen Beitrag zur Verwirklichung der Institutsziele leisten private Stiftungen. Das DPI hat satzungsgemäß die Aufgabe, durch seine Arbeit zur Vertiefung der gegenseitigen Kenntnisse des kulturellen, geistigen und gesellschaftlichen Lebens von Polen und Deutschen beizutragen.

Ziel der Vermittlertätigkeit des DPI ist es, »die zu interessieren, auf die es politisch, wirtschaftlich, gesellschaftlich und kulturell im deutsch-polnischen Verhältnis ankommt« (Leitlinien 1997). Es geht um die Entscheider und Multiplikatoren in Politik, Kultur, Bildung, Verwaltung, Medien und Wirtschaft und, wesentlich stärker ausgeprägt als bisher, um das Hineinwirken in Wissenschaft, Forschung und Bildung.

Derzeit bemüht sich das DPI in Kooperation mit den verstreuten Orten wissenschaftlicher Polen-Kompetenz an deutschen Hochschulen und Forschungsinstituten verstärkt darum, ausgehend von einer Bestandsaufnahme deutscher Polen-Forschung Ort wissenschaftlicher Forschung und verbindendes, vernetzendes und kooperierendes Zentrum zu werden. Ausgangspunkt der Neuausrichtung ist die kaum mehr kontrollierbare Dynamik des Rückbaus der Ressourcen der wissenschaftlichen Polen-Kompetenz in den unterschiedlichen Disziplinen. Mit der über 50.000 Bände zählenden multidisziplinären Fachbibliothek für Polen mit einer einzigartigen Sammlung polnischer Literatur in der Originalsprache und in deutscher Übersetzung ist das DPI bereits ein geschätzter Ort der Recherche und des wissenschaftlichen Arbeitens. (w w w.deutsches-polen-institut.de)

\section{Forschungsstelle Osteuropa an der Universität Bremen}

1982 gegründet, widmet sich die Forschungsstelle Osteuropa an der Universität Bremen kulturellen und gesellschaftlichen Entwicklungen der Länder Ost- und Ostmitteleuropas in Zeitgeschichte und Gegenwart. Die Forschungsstelle besitzt in ihrem Archiv eine einzigartige Sammlung alternativer Kulturgüter und unabhängiger Texte aus den ehemaligen sozialistischen Ländern. Darunter befindet sich auch eine umfangreiche Sammlung des "Zweiten Umlaufs«, die das Schrifttum und Dokumente unabhängiger Initiativen und gesellschaftlicher Gruppen in Polen aus der Zeit von 1976 bis zum Umbruch umfasst. Neben ausführlicher individueller Forschung zu Dissens und Gesellschaft im Sozialismus, leitet die Forschungsstelle seit Januar 2007 ein gemeinsames Projekt mit einem Verbund von internationalen Forschungsinstituten zum Thema "Das andere Osteuropa - die 1960er bis 1980er Jahre, Dissens in Politik und Gesellschaft, Alternativen in der Kultur. Beiträge zu einer vergleichenden Zeitgeschichte«, welches von der VolkswagenStiftung finanziert wird.

Im Bereich der post-sozialistischen Gesellschaften sind in den letzten Jahren umfangreiche Forschungsprojekte durchgeführt worden, deren Schwerpunkte auf politischen Entscheidungsprozessen, Wirtschaftskultur und der EU-Osterweiterung lagen. Eine der Hauptaufgaben der Forschungsstelle ist die Information der interessierten Öffentlichkeit. Dazu gehören unter anderem regelmäßige E-Mail-Informationsdienste mit fast 15.000 Abonnenten in Politik, Wirtschaft und den Medien.

Mit ihrer in Deutschland einzigartigen Sammlung von Publikationen zu Osteuropa ist die Forschungsstelle eine Anlaufstelle sowohl für Wissenschaftler als auch für die interessierte Öffentlichkeit. In der Bibliothek sind derzeit neben anderen breit angelegten Beständen allein aus Polen ca. 300 laufende Periodika zugänglich. Die Bestände werden in Datenbanken systematisch erfasst. (w w w.fo rschung sstelle.uni-b remen.de)

Die Meinungen, die in den Polen-Analysen geäußert werden, geben ausschließlich die Auffassung der Autoren wieder. Abdruck und sonstige publizistische Nutzung sind nach Rücksprache mit der Redaktion gestattet.

Redaktion: Prof. Dr. Dieter Bingen (Darmstadt), Dr. Stefan Garsztecki (Bremen), Silke Plate, M.A. (Bremen) Technische Gestaltung: Matthias Neumann

Polen-Analysen-Layout: Cengiz Kibaroglu, Matthias Neumann

ISSN 1863-9712 (c) 2008 by Deutsches Polen-Institut Darmstadt und Forschungsstelle Osteuropa, Bremen

Kontakt: Dr. Andrzej Kaluza, Presse- und Öffentlichkeitsarbeit, Deutsches Polen-Institut, Mathildenhöhweg 2,

D-64287 Darmstadt, Tel.: 06151/4985-13, Fax: 06151/4985-10, E-Mail: polen-analysen@dpi-da.de, Internet: www.laender-analysen.de/polen 